Rebecca Henn | Caldieron, Miller | Constance Bodurow Stallmeyer, Dearborn | Seth Wiley | Brian Szymanik

\section{Materials Testing - Digital Ecology}

\author{
Seth Wiley, Point B Design
}

\section{Introduction}

Access to credible building product $^{1}$ performance information throughout the design and construction process is critical to enable project development, vet product selections, ensure as-built quality, and successfully complete construction. This is common knowledge and part of common practice for nearly all parties involved in design and construction. ${ }^{2}$ The sources of such information can range from vernacular to formal - from common practice to special reference. The focus of this paper is one of the more formal or specialized information sources, performance testing, as well as how such performance testing information can be better used. This paper's goals are to familiarize the reader with performance testing and to depict a new kind of valuable informational tool (digital ecology). Reference to pertinent nomenclature, description of a real world example, and detailed description of such an informational tool's values will be provided.

The major content of this paper was developed during project-based work and firm-funded internal research at point $b$ design, Itd. over approximately the previous 4 years. The phrase 'digital ecology' as herein used is a new concept proposed by the author. The analysis contained in this paper could be applied to the field of operations and maintenance as it is herein applied to design and construction; however, operations and maintenance is beyond the scope of this paper and may be addressed in future papers. It is my hope that this paper will contribute to tangible and real improvements of the built environment via continued, positive development within academic and professional practice.

\section{Familiarization - Definitions \& Dynamics}

Performance testing can be divided into two main groups: standardized compliance tests (SCT) and research \& development tests (RDT). SCTs, in that they deal with standards compliance, are publicly established, documented, and understood; whereas RDTs, in that they predominantly deal with research \& development phases which come before marketplace deployment, are relatively private or non-public. ${ }^{3}$

SCTs verify performance to publicly agreed-upon safety values stipulated within nationally and internationally recognized codes and standards. Health, aesthetics, environmental, and other factors are the subject of such safety values. Performance to such values means legitimate compliance with such standards provided that such SCTs are performed by certified laboratories via stipulated protocols, with such certification and stipulation being issued by aforementioned national and international public authorities. SCTs are regularly conducted on new and existing materials, products, and assemblies with the results being published via prescribed means: publicly available standardized reports, product labeling, and others. And specific collections of specific compliance is regularly required by authorities having jurisdiction, owner's criteria, architectural specifications, and similar. ${ }^{4}$ This system is complex and nebulous but clearly documented. Refer below for an example of a typical SCT, NFPA-285.

In contrast to SCTs, RDTs are not strictly regulated. RDTs explore and ascertain building product performance to less universally or publicly recognized values and are often conducted via custom, non-standard protocols. $^{5}$ RDTs often fall outside the purview and scope of SCTs, and are mostly performed either in the development of new products and assemblies or to make progress toward successful SCTs. RDTs may be conducted at various locations such as third party testing laboratories (such as those which regularly conduct SCTs) or a manufacturers' own facilities. RDT locations, occurrences, protocols, conduct, and results are typically kept private by contracting entities for reasons of public perception, intellectual property, and trade secrecy. However, the conduct and purpose of RDTs often closely "orbits" SCTs to provide potentially efficient transition from building product development to marketplace availability. Refer below for an example of a RDT, small-scale NFPA-285 and Custom Burning Test.

Though $\mathrm{A} / \mathrm{E} / \mathrm{C}$ including legal, building science, and manufacturing professionals make up the broader community involved with SCTs and RDTs, Engineering, Construction, and Building Science persons are those primarily involved with the actual carrying out of test management, conduct, analysis, and reporting. RDTs and SCTs are composed of similar kinds of operations and results.

\section{Example}

During 2009 and 2010, point b design was contracted by a global manufacturer of building-grade phenolic panel cladding products. The manufacturer needed help for its planned US market expansion, and felt that point $b$ design could provide value to its expansion effort. The manufacturer viewed point $b$ design's architectural design-build 
experience and general knowledge as valuable. Point b design possessed knowledge and capabilities well-suited to this manufacturer's particular needs: it could be a single source, low overhead, agile entity able to deliver relevant professional architectural consultation together with specialty contracting. The contract required, in part, auditing the manufacturer's currently-held product compliance certifications (SCTs), updating said compliance, and providing input to the manufacturer's positioning efforts (in terms of building product offerings and internal compliance regime) relative to projected changes in the American marketplace. These tasks were to bolster the manufacturer's goals of more universal marketplace acceptability of its building cladding products and assemblies.

This project was relatively large in scope and unique in nature for point $b$ design. The project required the development and negotiation of custom contracts; the assembly of compliance, sales, technical, and distribution entities; analysis and development of new building product assemblies; oversight of building product testing; and timely achievement of certain compliance certifications. Those ultimately directly involved were the manufacturer, a national distributor, a national sales director, a code compliance expert, two testing laboratories, and point b design.

Point $b$ design played two roles in this effort: general partner and independent third party. In its role as general partner, point $b$ design worked with the manufacturer to co-developed an agile-type contract to keep the project's overhead low, keep costs closely linked to incremental milestones, and provide sufficient risk insulation to both parties. (This general framework was mimicked by point $b$ design in the structuring of sub-contractor contracts.) In its role as independent third party, point $\mathrm{b}$ design agreed to receive no additional compensation from any other involved or competing party, ensuring the manufacturer's sole interests were served.

The manufacturer had been producing building-grade phenolic panel cladding products for approximately 20 years, selling such products in multiple global markets for as long. The manufacturer and its products had a strong and longstanding reputation for quality, and had many completed projects of various scales and degrees of complexity. The manufacturer, its production operations, and products held various ISO, CE, ASTM and similar certifications due its market presence in six major continents. The manufacturer additionally had sufficient internal financial and personnel resources to ensure that potential new testing programs and related tasks could be addressed in a timely fashion. However, given the manufacturer's somewhat large size, it had yet to update its product compliance specifically relative to new US building code changes. Additionally, the manufacturer lacked an internal US code and compliance expert resource.

Point b design is a small business which encompasses two independently incorporated but collaborating entities: a professional architectural entity and a licensed contracting entity. The business typically employs a flexible, customer-oriented business model for the delivery of unique projects within architecture and allied fields. Additionally, it leverages its computational expertise and construction knowledge to operate on larger project scales than would be typically allowed by its very few staff. So, despite the unique nature of this contract and scope of services, point b design was sufficiently used to negotiating custom contracts.

The overarching goal of the compliance program as-developed by point $b$ design and accepted by the manufacturer was the achieving of International Code Council's (ICC's) certification appropriate to this manufacturer's products. Choosing to pursue overall ICC certification rather than discrete SCTs has many benefits in terms of cost, time, and effort. Achieving this goal first required selecting the appropriate Evaluation Service (ICC-ES) Acceptance Criteria (AC), which is AC-92 Acceptance Criteria for Polymer-Based and Polymer-Modified Exterior and Interior Wall Cladding. ${ }^{6}$ (Among various Acceptance Criteria, the AC-92 was selected because at the time of this project it was commonly used for such products - reasonably fitting such products' composition; however, depending on the date at which this paper is being read, the appropriate $\mathrm{AC}$ number and categorization may differ. Please refer to ICC-ES ${ }^{7}$. ) Along with the AC-92, other Acceptance Criteria such as AC85 and $A C-10$ relating to the process of achieving ICC certification had to be observed. Point b design developed an appropriate compliance program taking into account necessary tasks and persons.

Among the many SCTs required in AC-92, NFPA-2857 (Standard Fire Test Method for Evaluation of Fire Propagation Characteristics of Exterior Non-Load-Bearing Wall Assemblies containing Combustible Components) is the most challenging for this type of product. This test is inherently difficult given its size, cost, and assembly complexity, but there were specific challenges for this product. The NFPA-285 became the major focus of point $b$ design's contract.

NFPA-285 was uniquely challenging to this product in terms of product composition, test assembly, and product history.

In terms of product composition, kraft paper is one of the major materials in phenolic panels, and kraft paper (cellulosic material) is quite flammable. Flammability, of course, varies given factors such as additional materials present in the phenolic composite, levels of such additional materials, and manufacturing methods; nonetheless, fire tests are of significant concern for cellulose-based materials.

Test assembly was especially challenging for this product in this test. Phenolic panel cladding applications such as the one being discussed here typically create both vertical and horizontal cavities within the test assembly - providing potential passage of heat, hot air, and flame within the assembly - an inherent risk of failure. Additionally, this product assembly is made of many small parts - making it relatively complicated to coordinate and install for typical construction concerns. 
NFPA-285, also known as the Multi-story Test, tests an exterior envelope assembly (not a product alone). The certification is given to a tested product in a tested configuration, and must be installed as such in eventual field construction applications to preserve ICC compliance. This effectively creates a complicated situation because there can be many combinations of products in a cladding application, and one product manufacturer typically does not want to limit the marketplace's options for use of various substitution products. So, the limitations of the NFPA-285 protocol effectively bottleneck marketplace flexibility. Given this, the process of specifying all the secondary assembly products used in the NFPA-285 test assembly is complicated by vested concerns held by such secondary product's manufacturers. This shortfall can be partially counteracted by maintaining trade secrecy, but there are often completing interests which evolve.

Lastly, this product lacked a history of being tested on similar base-wall apparatuses. The product had achieved positive large-scale fire testing results in the past, but such test results were achieved using base-walls possessing much more advantageous heat absorption and dissipation characteristics - effectively improving the product assembly's potential to perform well.

In terms of making progress toward NFPA-285, there are no prescribed RDTs set out by NFPA-285, NFPA, AC-92, any other Acceptance Criteria, the ICC, any other building code, or by any other related entity. There are no prescribed performance data correlations between any SCTs or potential RDTs. [And again,] the set of SCTs as prescribed in ICC AC-92 as well as other $\mathrm{AC}$ are specifically designed as non-overlapping, testing for performances unique to each test. This makes data points acquired in any given SCT non-indicators of acceptable performance in any other given SCT. And, there are no detailed test assembly configurations stipulated in NFPA-285, only general test assembly sizing requirements and apparatus base-wall information.

The route to achieving NFPA-285 certification for such a product and assembly as this was therefore difficult. As such, developing custom RDTs (for their potential to define applicable performance relative to specific SCTs) present the only viable, constructive, incremental tools with which to make progress toward SCTs such as the NFPA-285. In terms of this example, a RDT (Custom Burning Test) was proposed to the manufacturer as a means to ascertain an understanding of how proposed products may perform given NFPA-285 conditions. Simply put, the process of this Custom Burning Test was to burn small samples of product similar to that which would be tested in the NFPA-285, tracking qualitative and quantitative burn data. Since any preexisting as well as in-process variables in product or test condition (including manufacturing defect, finish, color, thickness, material and air moisture, temperature, movement, etc.) could affect burn performance, all variables had to be tracked. Products samples were instrumented and tested with qualitative and quantitative temperature, time, and burn data recorded.
Products were burned at temperatures and with characteristics similar to NFPA-285 conditions. This was done so that there could be potential data point correlation between this Custom Burning Test and NFPA285. Throughout this process, marketplace acceptability was routinely referenced to ensure sales viability of final tested product and assembly. There was a general goal held by both the manufacturer and point $b$ that a RDT be performed which very closely mimicked the NFPA-285 - a RDT so similar to this SCT that there could be real data point correlation between the two. Such a RDT could all but ensure the product's passing of NFPA-285. And the larger goal of such a RDT would be to conserve the time, money, and effort involved in performing the actual NFPA285. So since the Custom Burning Test was quite small, testing product samples (not assemblies) of roughly 12"x12" size, a larger RDT was sought - falling between the Custom Burning Test RDT and the full-scale NFPA-285 SCT in terms of scale, coordination, and price.

Such a RDT presents a somewhat new approach to RDTs and SCTs so closely mimicking the associated SCT that it could practically predict SCT performance. Such a RDT as that which is mentioned here, Smallscale NFPA-285, effectively rarified the NFPA-285 assembly - reducing the configuration to its minimum instrumented size. As such, a RDT of this kind would save the client significant money, time, and effort for a variety of reasons - mainly by offering a way to best test for applicable performance without incurring costs of the actual SCT.

However, being such a new concept, and given that such solid product performance was established in the Custom Burning Test, this manufacturer decided to forego the Small-scale NFPA-285 RDT and move directly from the Custom Burning Test RDT to the actual NFPA285 SCT. One critical factor in this decision: the assembly proposed for NFPA-285 testing was sufficiently normative in configuration that the burn physics surrounding the assembly could be somewhat reasonably and easy assumed as similar to those present in the Custom Burning Test. The manufacturer's product and assembly passed NFPA-285.

\section{Future}

The focus of this paper is performance testing and how such performance testing information can be better used. The foregoing example together with its brief preceding familiarization is meant to give the reader a real world, firsthand perspective on some processes involved with performance testing. This and the following sections will depict how the author proposes that the process of performance testing can be better carried out and how the products of performance testing can be better used, as well as to whom such improvements may be valuable in what ways, including a discussion of this new kind of informational tool (digital ecology).

In the example above, it was one of point b design's internal goals to bring these two kinds of testing, SCT and RDT, into closer alignment - to save money, time, and effort for all parties involved in the process. 
(Specifically, it was and is the author's personal goal to understand what benefits and deficiencies may be held by bringing RDTs and SCTs into closer alignment.) Additionally, it was point $b$ design's internal goal to understand how such process improvements in could be valuable to others. We found that the Small-scale NFPA-285 RDT could be very useful to other potential manufacturers, designers, constructers, and owners given different testing goals beyond ICC AC-92; because, the Small-scale test is approximately $1 / 3$ the expense of the NFPA-285 and could provide similar performance confirmation. So if one's goal was an understanding of product performance rather than certified compliance, this test would be a great fit. A Small-scale NFPA-285 RDT could also be valuable to newer product assemblies which do not perfectly fit current compliance classification, because it could positively contribute to quantitative understanding of assembly performance without all the cost and complication of the actual NFAP-285 test; this Small-scale test could be done while/if ICC compliance classification were being established. The Small-scale test could also be very valuable to those with especially tight $r \& d$ testing budgets or to those building applications which significantly differ from those potentially accounted for in a Custom Burning Test or similar RDTs.

Traditionally, SCTs (and to a large extent RDTs) are viewed as discrete and specific. This is accurate and yet mistaken. Taking as example ICC ACs, for any given building product there is a set of required SCTs which must be enacted to evidence minimum performance to acceptable levels. Such SCTs are group-engineered to test for nonoverlapping performances (aka. each SCT tests for a certain kind of performance which no other SCT tests for). ${ }^{9}$ However, since the route to successfully completing SCTs can be somewhat non-linear with many RDTs performed, this multitude of RDTs can in fact hold some valuable overlapping performance data - thus creating ad hoc non-discreteness and relationship between SCTs. So in this way, the set of ICC-AC SCTs can be thought of as related and defining a kind of minimum set of RDTs.

RDTs, as with SCTs, can be developed to understand any given performance factor; however, there are less and more difficult tests relative in part to the product or assembly being tested. Fire performance testing is particularly difficult because burn is complex - a compound, non-scalable natural phenomenon which includes such associated complex phenomena as air movement, drying, ignition, etc. ${ }^{10,11}$ Further, the complex nature of burning can thus produce a surprising variety of effects in products and assemblies - with such effects being relatively difficult to analyze. This can be especially vexing given that, as an example, efficient product development desires strict quantification of test results along with linear, direct linkage between forensic test findings and product chemistry or assembly configuration. (Given this, fire testing is a prime example of the need to better link RDTs with SCTs - to improve potential efficiency of movement through compliance testing.) Nonetheless, even given the complex nature of burning, a relatively strict set of RDTs could be developed. This set of RDTs, as noted in the previous paragraph, could be developed through specific analysis of SCTs themselves - effectively backwards-engineering SCTs to their disaggregated, pared-down tested-for performances, with each of these disaggregated and pared-down performances tested-for through smaller-size RDTs (such as the Custom Burning Test). And even though data from such a set of RDTs may not be specifically correlate-able to associated SCTs, such RDT data could be reasonable indicators of SCT performance at a broader scale.

This would be extremely valuable for process or cultural reasons. The testing process is somewhat difficult. The expensive, technicallyoriented, and relatively complicated nature of SCTing makes it a difficult process for the clients which this author has spoken with. All parties involved desire to move most effectively through the required testing processes. More open, bilateral, and participatory discussion within the compliance testing process - drawing clients more closely into discussion with testing personnel - would better facilitate efficient process management and effective problem solving. In the experience of the author, the process of compliance testing can all-to-easily become overly focused on acquiring definitive, positive results far too early in the overall testing process. And this condition, being problematic to the efficient operation of the process, is often exacerbated by the lack of in-depth working relationships between parties involved. The problems just depicted are in part triggered by larger and complex SCTs because they are expensive and have many variables. So, having RDTs which either contain fewer complex phenomena or are small and cost less would partially alleviate one of impediments to efficient research and compliance testing. This would be a positive improvement to the culture of the process offered by bringing RDTs into alignment with SCTs, and it would benefit all persons involved.

RDTs could be brought into closer alignment with SCTs via a requirement that RDTs be reported-on in similar established public informational frameworks as those found in current SCT reporting. This would, by the very fact of making such RDT information available and useable, make research \& development information a more useable tool for design, construction, maintenance, etc. parties. In fact, [and again] there are RDTs carried out by building product manufacturers which test for factors beyond the strict scope of minimal building code requirements. So there is a kind of systemic inefficiency because such RDT information is not made available for use by other potential other parties such involved in design, construction, or maintenance entities. Such reporting could be included as additional information in documents such as ICC ESRs (International Code Council's Evaluation Service Reports). Or such information could be the basis for the creation of a new document - in either a new format or modeled on similar documents such as the ESR, CCR, or similar. ${ }^{12}$ Further of course, the information contained in such a document could be structured as compatible with IFC, COBie (Construction-Operations 
Building information exchange), SPie (Specifiers' Properties information exchange), and other information management protocols for maximized value and future-proofing. Such information would be extremely valuable to BIM project conduct environments which incorporate and rely on performance information for specification and other purposes.

Within the sphere of building product testing there are new movements toward test downsizing. ${ }^{13}$ These efforts bolster the continuous efforts to increase our understanding of building physics through the understanding of product performance. The ICC has also improved and expedited the process of obtaining ESRs, streamlining and clarifying the process. These changes come, in part, for economic reasons on the part of ICC and Testing Laboratories; and, they are beneficial to manufacturers, owners, architects, and contractors. An updated approach to testing information reporting such as one depicted in the preceding paragraph would fall in-line with and bolster such efforts.

Since most RDTs are typically performed en route to the positive completion of SCTs, the effective total set of RDTs is typically rarefied down to one closely resembling standardized compliance - with only those tests being performed which explicitly contribute to correlate-able SCT data points. This narrowing of the potential set of RDTs leaves a potential total set of quantitatively and qualitatively understood product performances drastically stunted and incomplete. Such factors as varying fire performance above and below prescribed standardized compliance values, material thermal and moisture expansion and contraction in non-standard applications, compound cold bending of planar materials, natural coloration and texture of building-grade composites, etc. are reasonable RDTs given current architectural practice, but they are not typically performed. If the starting point for the justification of testing gave greater significance to an expanded breadth of plausible architectural design applications, there would most likely be an expanded set of prescribed minimum SCTs. Further, if potential building applications of new products were required to be defined in part through an open and defined process of RDTs, the transition from research \& development into standardized compliance could be far easier and the breath of product innovation would increase. Additionally, if the funding entities for such RDTs were to differ from those typically in the current marketplace, the goals of such RDTing would most likely differ as well - changing the entire landscape of relationships between RDTs and SCTs.

There is another approach to improving efficiency between RDTs and SCTs which will be herein referred to as digital ecology (DE). DE will be defined as a matrix of varying cross-compatible materials / products I assemblies with qualitative and quantitative performance projections referenced to Acceptance Criteria levels. Such a DE is informational and could be developed via a calculated set of traditional engineering evaluations carried out on various similar materials / products / assemblies within, as an example, a given building system application category. The use of such a DE could be a detailed viability scenarioization of various building envelop assembly configurations including varying sub-component configurations. The results of such a $D E$ could be a hybrid broad and deep analysis for vetting of alternatives en route to best fitness for any given project, and identification of required associated specifications given a specific set of materials I products / assemblies. As such, conceptually, this would fit in with current notions of BIM, and the increasingly multidisciplinary disposition of all design stages. ${ }^{14}$ Such a digital ecology could be developed for panelized back-vented, back-drained envelop systems, giving weighted significance to girt material, gauge, and galvanization. It could be done for evaluation of initial construction costs, development typologies in varying US national locations, etc. Such an application of this DE would be beneficial because such envelop systems are commonly specified using unnecessarily expensive sub-components and hardware. So it would be useful to understand the viability of alternative products given implications for performance, cost, constructability, sustainability, etc. Further, many such envelops are designed and constructed with an inherent inefficiency of material overage and serviceable lifespan. This is overlooked in part because no such cross-reference matrix currently exists. Cost estimators and constructability experts are of course capable of carrying out portions of such work, but such a hybrid and flexible information tool is not available in either public, more generic, or uniform formats. Additionally, neither cost estimators nor constructability experts possess particular expertise in compliance, performance, and physics. This prevents such professionals from being able to deliver the function which such a DE would possess. Such DE matrix information, whether digitized or not, would be beneficial to many involved in building finance, ownership, design, construction, operation, maintenance, and [possibly even] deconstruction. However, the encoding of such information into a neural network such as that discussed in work of Gavalda et al. could present a kind of next-generation value for unintended but useful interlinkages within data point constellations. ${ }^{15}$

It would be relatively easy, inexpensive, and quick to have engineering evaluations performed to develop such a matrix, as engineering evaluations are typically far less expensive than related testing. This is especially the case relative to more costly SCTs such as NFPA-285. The requisite analysis and reporting fed into such a DE would rely on small amounts of strategic professional architectural thinking. Such critical thinking would focus on reasonable ranges of reasonable alternate constructions combined with a slightly larger amount of professional engineering evaluation of such alternatives.

\section{Professional Value}

This arena of material / product / assembly testing is currently handled predominantly by engineering and construction professionals. This paper is concerned with encouraging greater involvement by the Architectural discipline. The lack of quantifiable RDT capability taught and carried out 
by the architectural discipline is to its own disadvantage in terms of billing, facility with new design regulation, and forensic analysis. Architectural education provides cursory education of SCTs through courses such as Professional Practice. Intern or Professional development provides contact with SCT information via normal project conduct and document development. Nonetheless, there is a lack of in-depth education in SCTs in Architecture to develop such good working facility with SCTs that there could be wider professional involvement. There is really no Architectural education which relates specifically to RDTing. This is unfortunate and disadvantageous. Architects have a solid professional base of knowledge for involvement with this arena, and their involvement could provide increased value stemming from unique design perspectives on alternative material / product / assembly configurations and applications, as well as forensic analysis. Contracts for such work could be negotiated with manufacturers - some of the same manufacturers whom are already involved in architectural projects. Relationship to specifications, non-product-specific specifications, performance specifications, and the role of architect to push performance through specification of greater performance values are facets of architectural practice which could benefit from improved architectural education of and professional involvement with RDTs and SCTs.

RDTs and SCTs are developed via normal analytical frameworks and conducted through standard and established means in traditional testing laboratory facilities. Such conditions would seemingly facilitate morethen-current architectural engagement and involvement. Yet there are seemingly few architectural practitioners involved with SCTs and RDTs. Architectural design can be thought of as dealing with a kind of highlevel RDTs - new building applications developed through project-based designs which push the performance of various products to new levels. However, architectural practice does not get involved at a more detailed and consistent level in RDTing which would bolster the profession's base of building science knowledge founded on firsthand experience. If developed, such firsthand and in-depth knowledge could positively re-inform professional design. At the broadest scale, benefits could be reaped by all those inhabiting the built environment. More directly, improved understanding of materials / products / assemblies testing would improve the real quality of projects delivered to owners through such means as increased performance, reduced construction difficulties, and decreased cost for new project types.

As found in the example above, some involved in construction and building product manufacturing surprisingly do not have the luxury or fortune of expert building code knowledge. The ability for a manufacturer to contract with an architectural professional for code compliance and associated testing services can be a valuable service. Of course, the proposition that there be more popular adoption of such a new kind of service, as with any new potential business type, possess associated augmentations to traditional relationships, engagements, and contracts - expanding professional participation to potentially create new alignments of scale, industry, adjacency, and alliance. Some persons may differ in opinion, but this author identifies all such changes as positive because their goal is improvement of the built environment, improvement of process efficiency, and increased business potential.

Lastly, current given changing notions and requirements of environmental health, multi-disciplinarity, building performance, information exchange, and design excellence, the current standard of care used to develop and carry out material / product / assembly testing should be duly reexamined. Both governing bodies as well as related professional organizations should reexamine the cultural frameworks and associated expectations held by their own practitioners. These are professional ethical concerns as well as pragmatic issues.

\section{Conclusion}

Such a DE as proposed herein would benefit parties involved in design, construction, manufacturing, testing, and compliance - embodying efficiencies which could be passed on to owners and operators. Increased access to new compilations of performance information is a critical link in improving built environments. And the value of such performance information increases as projects become more challenging due to either internal or external constraint conditions. ${ }^{16}$

Among other things, this paper depicts an opportunity for innovation within the process of performance testing, and how such innovation could be valuable to many parties involved in the ownership, design, construction, and maintenance of the built environment. Additionally, this paper details how architecture can more effectively participate in the field of testing, and the value such participation can hold for the parties involved. Much of this paper may not be new thinking to the many practitioners, but some of the concepts held herein may be new to some. This paper's goal is to educate those unfamiliar with such testing, to demonstrate its value, and identify new useful ways of working in the field. Rationales for the improvement of work methods and results can be found in professional ethics, expansion of billable services, expanded design opportunities, increases efficiency, improved interdisciplinarity, improved technology and computation, and more. There are some relatively near-term projects, such as $D E$, which would improve the process and product of the built environment; however, encouraging a longer and broader move toward increased knowledge of and facility with our environment is the deep and (hopefully more fruitful) goal of this paper. 


\section{Endnotes}

1. For ease of reading, the phrase "building product" will be used to refer collectively to building products, materials and assemblies. Though there are substantial differences between these three items, they are closely related given this article's general focus. They will be referred to separately when required for topical discussion.

2. J.A. Demkin, ed., Architect's Handbook of Professional Practice, 13th ed. (John Wiley \& Sons, Inc., 2001), Pg. 505.

3. R. Bukowski, Y. Hirano, T. Rackliffe, R. Bowen, Standards Linkages to a Performance-based Regulatory Framework (Wellington, New Zealand: CIB World Building Congress, April 2001), Pg. 2.

4. J.A. Demkin, ed., Architect's Handbook of Professional Practice, 13th ed. (John Wiley \& Sons, Inc., 2001), Pg. 509.

5. See research and development testing resources from major testing laboratories such as Intertek, ATI, SWRI.

6. See ICC-ES website: www.icc-es.org/Criteria/pdf_files/ac92.pdf

7. See ICC-ES website: www.icc-es.org

8. See NFPA website: www.nfpa.org

9. Federation of American Scientists, Product Certification and Evaluation: A Comparison of Approaches to Building Product Approval.

10. A. Hamins, M. Bundy, Characterization of Candle Flames (Journal of Fire Protection Engineering, Vol. 15, Nov. 2005).

11. R. Feynman, Fun to Imagine 2: Fire (www.youtube.com).

12. See ATI CCR: http://www.archtest.com/certification/code_compliance_ research reports ccrr.aspx

13. P.N. Whiting, Development of the Vertical Channel Test Method for Regulatory Control of Combustible Exterior Cladding Systems (Branz, Study Report No. 137, 2005).

14. D.E. Ways, The Wave of the Future: AEC gTLD (Journal of Building Information Modeling, Spring 2011) Pg. 34.

15. X. Gavalda, J. Ferrer-Gener, G.A. Kopp, F. Giralt, Interpolation of pressure coefficients for low-rise buildings of different plan dimensions and roof slopes using artificial neural networks (Journal of Wind Engineering and Industrial Aerodynamics, Vol. 99, Is. 5, May 2011) Pg. 658-684.

16. B. Meacham, B. Tubba, D. Bergeron, F. Szigeti, Performance System Model - A Framework for Describing the Totality of Building Performance (Melbourne, Australian: 4th International Conference on Performance-Based Codes and Fire Safety Design Methods, March 20-22, 2002), Pg. 1. 